\title{
MODULES WITHOUT INVARIANT BASIS NUMBER
}

\author{
W. G. LEAVITT
}

In a recent paper, ${ }^{1}$ [p. 190] the author constructed a ring over which a finitely based module has invariant basis number if and only if it has a basis of length $<2$. It was indicated that this construction was generalizable, and this proceeds as follows:

Let $R$ be a word ring (with unit) in symbols $\left\{a_{i j}, b_{s t}\right\}(j, s=1, \cdots$, $n \geqq 2 ; i, t=1, \cdots, n+1)$ over the rationals. Let $A$ and $B$ be matrices whose elements are respectively $\left\{a_{i j}\right\}$ and $\left\{b_{s t}\right\}$, and consider the relations of

$$
A B-I_{n+1}=0, \quad B A-I_{n}=0 .
$$

It is clear that the method of proof [Lemma 1, p. 191] is applicable, and thus for each $\alpha \in R$ we may obtain in a finite number of steps a unique normal form $N(\alpha)$ not containing any of the leading words of the left-hand members of relations (1). If $H$ is the two-sided ideal whose basis is the set of all elements of $A B-I_{n+1}$ and $B A-I_{n}$, we accordingly have an effective means of deciding whether or not $\alpha \in H$ (namely $\alpha \in H$ if and only if $N(\alpha)=0$ ). The quotient ring $K=R / H$ may also be regarded as a word ring in $\left\{a_{i j}, b_{s t}\right\}$ all of whose members are reduced to normal form.

It may also be verified that $K$ contains no zero divisors, the proof following that of [Lemma 2, p. 192]. Note that if the degree $d[\alpha]$ is defined to be the length of the longest word in $\alpha$, this proof also shows that $d[\alpha \beta]=d[\alpha]+d[\beta]$. According to the remarks of [p. 193, footnote $]$ it is clear that a module over $K$ with a basis of length 1 has invariant basis number, while a module with a basis of length $\geqq n$ does not. This leaves the question open for a module over $K$ with basis of length $q(1<q<n)$. It is the purpose of the present paper to show that such a module also has invariant basis number.

A word $y$ is said to be similar to $x$ if it differs from $x$ only in either or both (a) the first subscript of its first symbol, (b) the second subscript of its last symbol; $y$ is left $\{$ right $\}$ similar to $x$ if only (a) $\{$ (b) $\}$ applies. Let $\left\{m_{\boldsymbol{i}}\right\}(i=1, \cdots, s)$ be a partition of the integer $m$. If $x$ is a word of length $m$, a word $y$ is said to be compatible with $x$ (relative to the partition $\left.\left\{m_{i}\right\}\right)$ if $x=x_{1} x_{2} \cdots x_{8}$ and $y=y_{1} y_{2} \cdots y_{s}$, where $d\left[x_{i}\right]=d\left[y_{i}\right]=m_{i}$ with $y_{1}$ right similar to $x_{1}, y_{8}$ left similar to $x_{s}$, and

Received by the editors May 10, 1956.

1 William G. Leavitt, Modules over word rings, Proc. Amer. Math. Soc. vol. 7 (1956) pp. 188-193. References to this paper will be enclosed in brackets. 
$y_{i}$ similar to $x_{i}(1<i<s)$. Note that if $u$ and $v$ are words (in normal form) such that $d[u]=\sum_{1}^{k} m_{i}$ and $d[v]=\sum_{k+1}^{s} m_{i}$, then if the product $u v$ is compatible with $x$ before normalization, all its longest words remain compatible after normalization. An $\alpha \in K$ is homogeneous if its words are all of the same length.

Suppose $\left\{\alpha_{i}, \beta_{i}\right\}(i=1, \cdots, q ; 1<q<n)$ is a set of homogeneous members of $K$ (in normal form) such that $d\left[\alpha_{1}\right] \geqq d\left[\alpha_{2}\right] \geqq \cdots \geqq d\left[\alpha_{q}\right]$ and such that for all $i$ we have $d\left[\alpha_{i} \beta_{i}\right]=m$. Suppose further that all longest words in any of the products $\alpha_{i} \beta_{i}$ are compatible relative to the partition of $m$ given by the nonzero numbers in the sequence

$$
d\left[\alpha_{q}\right], d\left[\alpha_{q-1}\right]-d\left[\alpha_{q}\right], \cdots, d\left[\alpha_{1}\right]-d\left[\alpha_{2}\right], d\left[\beta_{1}\right] .
$$

LEMma 1. If the above set $\left\{\alpha_{i}, \beta_{i}\right\}$ also satisfies the condition $d\left[\sum \alpha_{i} \beta_{i}\right]<m$, then (possibly relabeling those $\alpha_{i}$ for which $d\left[\alpha_{i}\right]$ $\left.=d\left[\alpha_{1}\right]\right)$ there exist $\left\{\theta_{i}\right\}(i=2, \cdots, q)$ such that $d\left[\alpha_{1}+\sum_{2}^{q} \alpha_{i} \theta_{i}\right]$ $<d\left[\alpha_{1}\right]$.

If any $\alpha_{k}$ is constant, the lemma follows trivially, for we may take $\theta_{k}=-\alpha_{1} / \alpha_{k}, \theta_{i}=0(i \neq k)$. Similarly, if $\beta_{1}$ is constant we may choose $\theta_{i}=\beta_{i} / \beta_{1}$. Accordingly we suppose that all $d\left[\alpha_{i}\right], d\left[\beta_{i}\right]>0$.

By compatibility the words of any particular $\alpha_{r}$ all end with either $a_{p i}$ or $b_{p i}$ (for fixed $p$ ), those of $\beta_{r}$ all begin with either $a_{j q}$ or $b_{j q}$ (for fixed $q$ ). Call a product $\alpha_{r} \beta_{r}$ of type $a b$ if the words of $\alpha_{r}$ end in $a_{p_{i}}$ while those of $\beta_{r}$ begin with $b_{j q}$. A similar description applies to products of type $b a, a a$, or $b b$.

Let us suppose that the partition of $m$ mentioned above has $s$ members, then also by compatibility the words of $\alpha_{1}$ have in general $2 s-3$ variable indices, while those of $\beta_{1}$ have a single variable index. Call these indices $i, \cdots, j$ and $k$. If $d\left[\alpha_{1}\right]=\cdots=d\left[\alpha_{t}\right]>d\left[\alpha_{t+1}\right]$ $\geqq \cdots$, this also applies to all $\alpha_{r}, \beta_{r}$ for which $1 \leqq r \leqq t$. Let the coefficient of the word (of such an $\alpha_{r}$ ) with variable indices $i, \cdots, j$ be $c_{i}^{r} \ldots j$, and of the word of $\beta_{r}$ with index $k$ be $d_{k}^{r}$. If $\alpha_{r} \beta_{r}$ is a product of type $a b$ or $b a$, the coefficients of its longest words are $c_{i}^{r} \ldots j d_{k}^{r}$ $-\delta_{j k} c_{i}^{r} \ldots 1 d_{1}^{r}$ (where $\delta_{j k}=1$ if $j=k, 0$ otherwise), while if the product is of type $a a$ or $b b$ the second term is omitted. Proceeding to those $\alpha_{r}$ for which $d\left[\alpha_{r}\right]=d\left[\alpha_{t+1}\right]<d\left[\alpha_{t}\right]$, the words of such an $\alpha_{r}$ have $2 s-5$ variable indices $i, \cdots$ and those of the corresponding $\beta_{r}$ have three - $j k$. If such an $\alpha_{r} \beta_{r}$ is a product of type $a b$ or $b a$ its longest words have coefficients $c_{i}^{r} \ldots d_{\cdot{ }_{j k}}^{r}-\delta . . c_{i}^{r} \ldots 1 d_{1 j k}^{r}$ where the subscripts of $\delta .$. are the last of $c_{i}^{r} \ldots$ and the first of $d^{r} \cdot{ }^{r}$. Again, if the product is of type $a a$ or $b b$, the second term is omitted. Similar expressions are evidently obtained for the coefficients of the longest words of all $\alpha_{i} \beta_{i}(i=1, \cdots, q)$.

Now $q<n$ and each variable index has range either 1 to $n$ or 1 to 
$n+1$, so each variable index has at least $q+1$ values. Also $d\left[\alpha_{q}\right]>0$, so at least one $c_{i}^{q} \neq 0$ (say, for definiteness, it is among $c_{2}^{q}, \cdots, c_{q+1}^{q}$ ). We now choose, for the moment, the subscripts $i, \cdots, j$ as follows: the second and succeeding subscripts will be fixed, with the second equal to 1 , the last $q+1$, and no two adjacent subscripts equal. Since $d\left[\alpha_{1} \beta_{1}+\cdots+\alpha_{q} \beta_{q}\right]<m$, it follows that all the coefficients of its longest words are zero. Thus, with the above choice of subscripts, we have

$$
\stackrel{1}{c_{i 1} \cdots q+1} d_{k}^{1}+\cdots+c_{i}^{q} d_{1}^{q} \cdots_{q+1 k}=0,
$$

where we choose $i=2,3, \cdots, q+1$ and $k=1, \cdots, q$.

Since at least one of the $c_{i}^{q} \neq 0$, the rank of the coefficient matrix of (3) is at least one. Thus the set of vectors $\left(d_{k}^{1}, \cdots, d_{1}^{q} \cdots_{q+1 k}\right)$ is dependent. We distinguish the following cases: ${ }^{2}$

Case I. $d_{1}^{1}=\cdots=d_{1}^{t}=0$. Since $d\left[\beta_{1}\right]>0$, at least one, say $d_{\mathfrak{u}}^{1} \neq 0$. Then for any choice of the subscripts $i, \cdots, j$ we have

$$
\begin{gathered}
c_{i}^{1} \ldots d_{u}^{1}+\cdots+ \\
+c_{i}^{t} \ldots d_{u}^{t}+c_{i}^{t+1} d_{\cdot j u}^{t+1}-\delta . . c_{i \cdot 1}^{t+1} d_{1 j u}^{t+1}+\cdots \\
+c_{i}^{q} d_{\ldots j u}^{q}-\delta_{i} . c_{1}^{q} d_{1}^{q} \ldots j u=0 .
\end{gathered}
$$

We choose $\theta_{r}=d_{u}^{r} / d_{u}^{1}$ for $(r \leqq t)$, while for $r>t$ we form the words of $\theta_{r}$ by dropping from the words of $\beta_{r}$ the portions similar to $\beta_{1}$. If the variable subscripts of a resulting word are $\cdots j$, we choose for its coefficient $d^{r} \ldots j u / d_{u}^{1}$ (that is, the coefficient of $c_{i}^{r} \ldots$ in the equation derived from (4) by dividing by $\left.d_{u}^{1}\right)$.

Case II. Some $d_{1}^{h} \neq 0$ (by relabelling, this becomes $d_{1}^{1} \neq 0$ ) and for some $u$ all $d_{u}^{1}=\cdots=d_{u}^{t}=0$. Then for any choice of subscripts $i, \cdots, j$ we have

$$
\stackrel{1}{c_{i} \ldots j} d_{1}^{1}+\cdots+c_{i}^{t} \ldots{ }_{j} d_{1}^{t}+\cdots+c_{i}^{q} d_{\cdots j 1}^{q}-\delta_{i} \cdot c_{1}^{q} d_{1}^{q} \ldots j 1=0
$$

$$
-c_{i}^{1} \ldots{ }_{1} d_{1}^{1}-\cdots-c_{i}^{t} \cdots_{1} d_{1}^{t}+\cdots+c_{i}^{q} d_{\cdots u u}^{q}-\delta_{i} \cdot c_{1}^{q} d_{1}^{q} \cdots u u=0 .
$$

Again $\theta_{r}=d_{1}^{r} / d_{1}^{1}(r \leqq t)$, and for $r>t$ the coefficients of $\theta_{r}$ (with subscripts $\cdots j)$ are $d^{r} \ldots{ }_{11} / d_{1}^{1}$ if $j \neq 1$ and $-d^{r} \ldots u u / d_{1}^{1}$ if $j=1$.

Case III. For each $k$ at least one of $d_{k}^{1}, \cdots, d_{k}^{\mathfrak{k}} \neq 0$. Since the vectors $\left(d_{\mathbf{k}}^{1}, \cdots, d_{1}^{q} \cdots q+1 k\right)$ are dependent, there must be some $u$ such that $\left(d_{\mathfrak{u}}^{1}, \cdots\right)=\sum_{\mathbf{k}=1}^{u-1} h_{k}\left(d_{\boldsymbol{k}}^{1}, \cdots\right)$ for some set of constants $\left\{h_{k}\right\}$.

2 Remark that we are considering the worst situation, namely that in which all of the products $\alpha_{i} \beta_{i}$ are of type $a b$ or $b a$. It is clear that a similar, though simpler, treatment may be used when some of the products are of type $a a$ or $b b$. This is especially so if $\alpha_{1} \beta_{1}$ is of this type, for then we have essentially only the following Case I. 
By relabelling, let $d_{u}^{1} \neq 0$. Then

(6) $\quad c_{i}^{1} \ldots j_{j} d_{u}^{1}+\cdots+c_{i}^{t} \ldots j_{j} d_{u}^{t}+\cdots+c_{i}^{q} d_{\cdots, j u}^{q}-\delta_{i} \cdot c_{1}^{q} d_{1 \cdots j u}^{q}=0$

$$
\begin{array}{r}
c_{i} \ldots u d_{k}^{1}+\cdots+c_{i}^{t} \ldots u d_{k}^{t}+\cdots+c_{i}^{q} d_{\cdots u k}^{q}-\delta_{i} \cdot c_{1}^{q} d_{1}^{q} \cdots u k=0 \\
(k=1, \cdots, u-1) .
\end{array}
$$

If the equations of (7) are multiplied respectively by $h_{k}$ and summed, the result is

(8) $c_{i}^{1} \ldots d_{u} d_{u}^{1}+\cdots+c_{i}^{t} \ldots u d_{u}^{t}+\cdots+c_{i}^{q} e^{q} \ldots u u-\delta_{i} \cdot c_{1}^{q} e_{1}^{q} \ldots u u=0$,

where $e^{r} \ldots u u=\sum_{\substack{u=1 \\ k=1}} h_{k} d^{r} \ldots u k$. Once again $\theta_{r}=d_{u}^{r} / d_{u}^{1}(r \leqq t)$ and the coefficients of the words of $\theta_{r}(r>t)$ are $d^{r} \ldots$ ju $/ d_{u}^{1}$ (if $\left.j \neq u\right)$ or $e^{r} \ldots u u / d_{u}^{1}$ (if $j=u$ ).

It is clear from (4), (5), or (6) and (8) (depending on which of the three cases applies), the above choice of $\theta_{r}$ gives coefficients of the longest words of $\sum \alpha_{r} \theta_{r}$ which are just sufficient to cancel the coefficients $c_{i}^{1} \ldots, j$ of the words of $\alpha_{1}$.

We now define an elementary transformation to be either a permutation (relabeling) $\left\{\alpha_{i}, \beta_{i}\right\} \rightarrow\left\{\alpha_{j}, \beta_{j}\right\}$ or a transformation of type $\left\{\alpha_{i}, \beta_{i}\right\} \rightarrow\left\{\alpha_{i}^{\prime}, \beta_{i}^{\prime}\right\}$ where for somer

$$
\begin{aligned}
\alpha_{r}^{\prime}=\alpha_{r}+\sum_{r+1}^{q} \alpha_{k} \theta_{k}, & \beta_{i}^{\prime}=\beta_{i} & & (i \leqq r), \\
\alpha_{i}^{\prime}=\alpha_{i}(i \neq r), & \beta_{i}^{\prime}=\beta_{i}-\theta_{i} \beta_{r} & & (i>r) .
\end{aligned}
$$

Lemma 2. Let $\left\{\alpha_{i}, \beta_{i}\right\}(i=1, \cdots, q<n)$ be members of $K$ arranged so that $d\left[\alpha_{i}\right] \geqq d\left[\alpha_{i+1}\right]$. If $m=\max d\left[\alpha_{i} \beta_{i}\right]$ and $d\left[\sum \alpha_{i} \beta_{i}\right]<m$, then there exist $\left\{\alpha_{i}^{*}, \beta_{i}^{*}\right\}$, reached by a finite sequence of elementary transformations, such that $\sum \alpha_{i}^{*} \beta_{i}^{*}=\sum \alpha_{i} \beta_{i}$ and $\max d\left[\alpha_{i}^{*} \beta_{i}^{*}\right]<m$.

Since the case $q=1$ is impossible, it is sufficient to show that from case $q$ follows either a case $\leqq q-1$ or the lemma. If for any $i$ we have $d\left[\alpha_{i} \beta_{i}\right]<m$, then we already have a case $\leqq q-1$. Thus suppose all $d\left[\alpha_{i} \beta_{i}\right]=m$. We again use the partition (2) determined by the $d\left[\alpha_{i}\right]$ and $d\left[\beta_{i}\right]$. Let $x$ be a longest word of $\alpha_{1}$ and $y$ of $\beta_{1}$, and let $\bar{\alpha}_{1}$ be the part of $\alpha_{1}$ containing all (and only) words compatible with $x$ relative to this partition. Similarly, $\bar{\beta}_{1}$ is the part of $\beta_{1}$ compatible with $y$. In general, $\bar{\alpha}_{i}$ is the part of $\alpha_{i}$ compatible with $x$ or a first portion of $x$, while $\bar{\beta}_{i}$ is the part of $\beta_{i}$ compatible with $u y$, where $u$ is either 1 or a last portion of $x$. Clearly, all longest words of $\bar{\alpha}_{i} \bar{\beta}_{i}$ are compatible with $x y$, and are not combinable with any other word 
obtained from any product $\alpha_{i} \beta_{i}$. Thus $d\left[\sum \bar{\alpha}_{i} \bar{\beta}_{i}\right]<m$, while $d\left[\bar{\alpha}_{i} \bar{\beta}_{i}\right]$ $=m$ for all $i$, and so the set $\left\{\bar{\alpha}_{i}, \bar{\beta}_{i}\right\}$ satisfies the conditions of Lemma 1. Hence (possibly after a permutation) there exist $\left\{\theta_{i}\right\}$ such that $d\left[\bar{\alpha}_{1}+\sum_{2}^{q} \bar{\alpha}_{i} \theta_{i}\right]<d\left[\bar{\alpha}_{1}\right]$. Now let

$$
\begin{array}{ll}
\alpha_{1}^{\prime}=\alpha_{1}+\sum_{2}^{q} \alpha_{i} \theta_{i}, & \beta_{1}^{\prime}=\beta_{1}, \\
\alpha_{i}^{\prime}=\alpha_{i}(i \neq 1), & \beta_{i}^{\prime}=\beta_{i}-\theta_{i} \beta_{1} \quad(i>1),
\end{array}
$$

then $\sum \alpha_{i}^{\prime} \beta_{i}^{\prime}=\sum \alpha_{i} \beta_{i}$, and all words compatible with $x$ have been eliminated from $\alpha_{1}$.

This means that $\alpha_{1} \beta_{1}$ (dropping primes henceforth, for convenience) now contains no word compatible with $x z$ for any word $z$ (with $d[z]=d[y])$. Thus if now $\sum_{1}^{q} \alpha_{i} \beta_{i}$ contains such a word, equating its coefficient to zero will involve nothing from $\alpha_{1} \beta_{1}$. We consider first the case $d\left[\alpha_{2}\right]=d\left[\alpha_{1}\right]$. If $\alpha_{2}$ contains a word compatible with $x$, either $d\left[\alpha_{2} \beta_{2}\right]<m$, in which case the lemma is proved, or all words compatible with $x$ may be eliminated from (possibly a permuted) $\alpha_{2}$ in exactly the manner above. This process may clearly be extended to eliminate $x$ from all words $\alpha_{i}(i=1, \cdots, t)$ for which $d\left[\alpha_{i}\right]=d\left[\alpha_{1}\right]$.

Now suppose $d\left[\alpha_{t+1}\right]<d\left[\alpha_{1}\right]$ and $x=u v$, where $d[u]=d\left[\alpha_{t+1}\right]$. If $\alpha_{t+1}$ contains a word compatible with $u$, while $\beta_{t+1}$ contains a word compatible with $v z$ (for any $z$ ), equating to zero coefficients of words in $\sum \alpha_{i} \beta_{i}$ compatible with $x z$ again involves nothing from $\alpha_{i} \beta_{i}(i \leqq t)$. Thus, in a similar way, $u$ may be eliminated from (a possibly permuted) $\alpha_{t+1}$. Thus we have (or could obtain) a situation in which either $\alpha_{t+1}$ contains no word compatible with $u$, or $\beta_{t+1}$ contains no word compatible with $v z$. Clearly this process may be continued until for each $i$, either $\alpha_{i}$ contains no word compatible with $u_{i}$, or $\beta_{i}$ contains no word compatible with $v_{i} z$ (where $x=u_{i} v_{i}$ and $d\left[u_{i}\right]=d\left[\alpha_{i}\right]$ ). Thus all words compatible with $x z$ have been eliminated from all $\alpha_{i} \beta_{i}$.

We now proceed by induction. We suppose that for a given $r$ for which $d\left[\alpha_{r+1}\right]<d\left[\alpha_{r}\right]$, for any word $u$ such that $d[u]=d\left[\alpha_{r}\right]$ we are able to perform a series of elementary transformations such that no word of $\alpha_{1}, \alpha_{2}, \cdots, \alpha_{r}$ begins with a word compatible with $u$, and if $u=u_{i} v_{i}$ with $d\left[u_{i}\right]=d\left[\alpha_{i}\right](i>r)$ for each $i$, either $\alpha_{i}$ contains no word compatible with $u_{i}$ or no word of $\beta_{i}$ begins with a word compatible with $v_{i}$.

Now suppose $d[h v]=d\left[\alpha_{r}\right]$ and $d[h]=d\left[\alpha_{r+1}\right]$, and we carry out the elimination described in the induction hypothesis. If $h w$ is another such word, we wish to show that such an eliminition relative to hw does not destroy that already accomplished relative to $h v$. The trans- 
formations are of the type $\alpha_{j} \rightarrow \alpha_{j}+\sum_{j+1}^{q} \alpha_{i} \theta_{i}$ and $\beta_{i} \rightarrow \beta_{i}-\theta_{i} \beta_{j}(i>j)$, for some fixed $j$. If $j \leqq r$ we know that no $\alpha_{i}(i \leqq r)$ begins with a word compatible with $h v$, so could not restore such a word to $\alpha_{j}$. Also for $i>r$ either (I) $\alpha_{i}$ contains no word compatible with the first part of $h v$ or (II) $\beta_{i}$ contains no word whose first part is compatible with the final part of $h v$. But in forming the words of $\theta_{i}$ we take the words of $\bar{\beta}_{i}$ and lop off the part compatible with $\bar{\beta}_{j}$. Since the remaining part is at least as long as the final part of $h v$, it follows that if (II) applies to $\beta_{i}$ then it also applies to $\theta_{i}$. Thus in either case $\alpha_{i} \theta_{i}$ cannot restore a word whose first portion is compatible with $h v$. Also, for $i>r$, if $\beta_{i}$ contains no word whose first part is compatible with the last of $h v$, this is clearly also true of $\beta_{i}-\theta_{i} \beta_{j}$.

Now when $j>r$, we are engaged in removing from $\alpha_{j}$ words compatible with $h$ or a first portion of $h$. Thus no such word would be restored to $\alpha_{j}$. Furthermore, for any $j>r$ for which such elimination is to be performed, the corresponding $\beta_{j}$ cannot contain a word whose first portion is compatible with the end of $h v$. Thus if $\beta_{i}$ has no such word, $\beta_{i}-\theta_{i} \beta_{j}$ cannot.

We can thus eliminate as described above, all words beginning with a word compatible with $h w$, for any $w$, from all $\alpha_{i}(i \leqq r)$; that is, all words beginning with a word compatible with $h$. Also if $\alpha_{r+1}$ has a word compatible with $h$, then $\beta_{r+1}$ has no longest word beginning with any $w$. This would mean $d\left[\alpha_{r+1} \beta_{r+1}\right]<m$ and the lemma would follow. Thus suppose $\alpha_{r+1}$ has no word compatible with $h$, and similarly for the remaining $\alpha_{i}$ for which $d\left[\alpha_{i}\right]=d\left[\alpha_{r+1}\right]$. Finally, if $h=h_{i} w_{i}$ with $d\left[h_{i}\right]=d\left[\alpha_{i}\right]$ (and $d\left[w_{i}\right] \neq 0$ ), either $\alpha_{i}$ has no word compatible with $h_{i}$ or $\beta_{i}$ has no word whose first part is compatible with $w_{i} w$ for any $w$. This establishes the induction.

By induction, then, for any $g$ for which $d[g]=d\left[\alpha_{q}\right]$, we may eliminate all words beginning with a word compatible with $g$ from $\alpha_{i}(i=1, \cdots, q)$. Clearly no further elimination can restore such a word to any $\alpha_{i}$ and hence we may eventually eliminate all longest words from some $\alpha_{i}$. Then $d\left[\alpha_{i} \beta_{i}\right]<m$ and the lemma follows.

Lemma 3. If $\sum_{1}^{q} \alpha_{i} \beta_{i}=1$ then there exists a finite sequence of elementary transformations to $\left\{\alpha_{i}^{+}, \beta_{i}^{+}\right\}$such that $\alpha_{1}^{+}$is a constant and $\alpha_{i}^{+}=0(i \geqq 2)$.

By a permutation $\sum \alpha_{i} \beta_{i}$ may be placed in condition to apply Lemma 2 , so there exists $\sum \alpha_{i}^{*} \beta_{i}^{*}=1$ such that $\max d\left[\alpha_{i}^{*} \beta_{i}^{*}\right]$ $<\max d\left[\alpha_{i} \beta_{i}\right]$. This may be continued as long as $\max d\left[\alpha_{i} \beta_{i}\right]>0$ (dropping ${ }^{*}$ for convenience). But suppose $\max d\left[\alpha_{i} \beta_{i}\right] \leqq 0$. Since $\sum \alpha_{i} \beta_{i}=1$, at least one $\alpha_{i} \beta_{i}=$ constant. By permutation we get $\alpha_{n}=k$ 
and we can eliminate all other $\alpha_{i}$. Another permutation gives $\alpha_{1}=k$, $\alpha_{i}=0$ (all $i \geqq 2$ ).

THEOREM. For each $n>1$ there exists a ring without zero divisors over which a finitely based module has invariant basis number if and only if it has a basis of length $<n$.

According to previous remarks we need consider only case $n>2$ and we need only prove that a module with basis of length $q<n$ has invariant basis number. This will be true if for any $m$ by $q$ and $q$ by $m(m>q)$ matrices $P$ and $Q$, the relation $P Q=I_{m}$ is impossible.

Suppose $P Q=I_{m}$. The first row of $P$ and first column of $Q$ satisfies Lemma 3. Clearly a nonsingular matrix $T$ exists such that the first row of $P^{\prime}=P T$ and first column $Q^{\prime}=T^{-1} Q$ receive any desired elementary transformation. Since the first row of $P^{\prime}$ is $\left(k_{1}, 0 \cdots 0\right)$ and $P^{\prime} Q^{\prime}=I_{m}$, it follows that the first row of $Q^{\prime}$ is $\left(1 / k_{1}, 0 \cdots 0\right)$. By a similar process, applied to the second row and second column of $P^{\prime}$ and $Q^{\prime}$, we reach $P^{\prime \prime}, Q^{\prime \prime}$ whose second rows are all zero except for the first two elements. But by this process we would reach $P^{*} Q^{*}=I_{m}$, where the last $m-q$ columns of $Q^{*}$ are zero.

UNIVERSITY OF NEBRASKA 